\author{
Neam F Agha ${ }^{1}$ \\ BDS, MSc (Lect)
}

Mahmood Kh Ahmed BDS, MSc (Assist Lect)

Dina A Al-Khashab2 BDS, MSc (Dent Special)

\section{Mesiodistal crowns angulation in Class II division 1 malocclusion}

\author{
1Dept of Pedod, Orthod and Prev Dent \\ College of Dentistry, University of Mosul
}

\author{
${ }^{2}$ Ninevah Health Directorate \\ Ministry of Health
}

\begin{abstract}
Aims: To evaluate the mesiodistal angulation of the crowns in 50 study models ( 25 for each sex) of Class II division 1 malocclusion and compare it to 38 study models (19 for each sex) of Class I normal occlusion to find the differences between the two groups so as to provide valuable information in the treatment of Class II division 1 malocclusion. Materials and Methods: Mesiodistal crown angulation is measured as the angle formed between the long axis of the crown and a line perpendicular to the occlusal plane. The data subjected to statistical analysis at $p<0.05$. Descriptive statistics (mean, standard deviation, minimum, maximum), correlation for all the crowns and comparison between right and left side, male and female and between Class I and Class II were carried out. Results: Significant differences in the angulation of the crowns between the two classes in the buccal segment of the upper dental arches for both sexes with significant higher values in Class II division 1 were found. For the lower arch there were significant differences in central, lateral incisors and second premolar in males and in all crowns except first molar in females with significant higher values in Class II division 1 indicating that the crowns are more mesially inclined in Class II division 1 malocclusion. Conclusion: The crowns were more mesially inclined for Class II division 1 malocclusion than for Class I occlusion. There was a correlation among teeth in each quadrant.
\end{abstract}

Key Words: Mesiodistal, angulation, Class II division 1.

Agha NF, Ahmed MKh, Al-Khashab DA. Mesiodistal crowns in Class II division 1 malocclusion. AlRafidain Dent J. 2006; 6(1): 53-63.

Received: 18/7/2005 Sent to Referees: 24/7/2005

Accepted for Publication: 10/11/2005

\section{INTRODUCTION}

Class II division 1 malocclusion represents the second most common type of malocclusion after crowding ${ }^{(1-4)}$ and it is the most frequently seen skeletal disharmony in orthodontic population. ${ }^{(5)}$

In Iraq a study carried out in Mosul City showed that Class II malocclusion represents $18.7 \%$ of the population ${ }^{6}$ while other study performed on rural community showed that $10.4 \%$ of the population had Class II malocclusion. ${ }^{(7)}$

It is characteristic for the teeth to have varying degrees of mesial inclination, the gingival portion of the long axis of the crown more distal than the incisal porti$\mathrm{on}^{(8,9)}$ and it is one of the means by which they are kept in tight contact. ${ }^{(10)}$
Each non orthodontic normal model had a distal inclination of the gingival portion of each crown. It varied with each tooth type, but within each type the tipping pattern was consistent from individual to individual. ${ }^{(8)}$

Normal occlusion is dependent upon proper distal crown tipping especially of the upper anterior teeth. Since rectangle occupies a wider space when tipped than when upright, thus the degree of tipping determines the amount of mesiodistal space that they consume and therefore, has a considerable effect on posterior occlusion as well as anterior esthetic ${ }^{(8)}$ and arch length. ${ }^{(11)}$

In orthodontics, angulation of the teeth is an important part of alignment of tee- 
$\mathrm{th}^{(12)}$ normalizing occlusion involves many factors that include normalizing mesiodistal angulation and labiolingual inclination of the teeth. These arrangements are crucial in closing and consolidating the interdental spaces and obtaining an ideal overbite-over jet relationship. ${ }^{(11)}$

On completion of orthodontic treatment, it is common practice to asses mesiodistal angulation of the teeth in the dental arches. This is especially important in case in which extraction of dental units was performed as part of treatment with these spaces being subsequently closed by orthodontic means, ${ }^{(13)}$ and normal angulation and inclination of the teeth were better achieved with the Roth appliance. ${ }^{(14)}$

Several methods may be used to assess the mesiodistal angulations of the teeth including direct assessment of the axial inclination of the clinical crowns but it offers limited information for diagnosing dental irregularities, ${ }^{(15)}$ some researchers used study cast which is the only method that can provide three dimensional reproduction of the dentition, ${ }^{(16)}$ radiographic techniques were also used to determine the axial inclination of the teeth including intraoral periapical radiograph, ${ }^{(17)}$ extra oral oblique cephalogram, ${ }^{(18)}$ and the orthopantomograph could be used. . $^{(9,19)}$

This study was directed to evaluate mesiodistal crowns angulation in Class II division 1 malocclusion, to compare these angulations with those of Class I normal occlusion, to assess the correlation coefficient between the measured values of the mesiodistal crown angulation for both upper and lower dental arches in males and females and also to know how much mesiodistal angulation required for correction during or after retraction in such malocclusion.

\section{MATERIALS AND METHOD}

The sample consisted of 50 dental models of Class II division 1 malocclusion, 25 for each sex, with bilateral Class II molar and canine relationships and over jet more than $5 \mathrm{~mm} .^{(20,21)}$ The models were taken from patients aged 13-16 years. Thirty eight dental models of Class I normal occlusion selected from the private clinics,
19 for each sex, with bilateral Class I molar and canine relationships and normal over jet and overbite $2-4$ mm. ${ }^{(22,23)}$ In both groups, the models with well aligned full complement of permanent dentition includeing incisors, canines, premolars and fist molars.

The method of measuring mesiodistal crowns angulation was done by placing the occlusal plane of the study model on the dental surveyor, then marking the center of cervical line for all the teeth and the center of incisal edge for anterior teeth and the center of occlusal plane for posterior teeth. Then connecting the two center points by a line which represent the long axis of the crown ${ }^{(8,12)}$ (Figure 1).

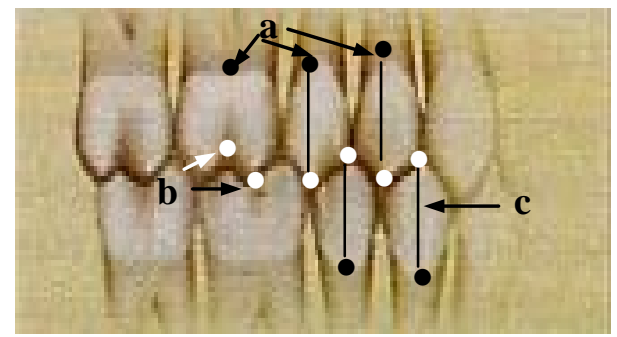

Figure (1): The long axis of the crown

A: Center of cervical line, b: Center of occlusal plane of posterior teeth, c: The long axis of the tooth

The amount of crown angulation recorded by the protractor (Figure 2) after adjusting the surveyor parallel to the horizontal plane and measure the mesiodistal angulation in which one arm of geometric device fixed on the plate of surveyor and the vertical line of the other arm (moveable arm) place on the long axis of the crown (Figure 3).






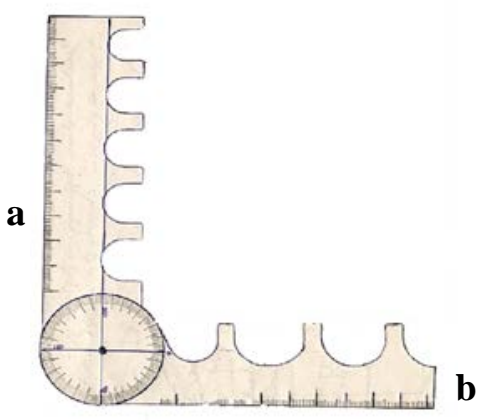

Figure (3): The protractor used in the study

A: Movable arm, b: Fixed arm

The records were expressed as positive (+ve) which means mesially inclined crown or negative $(-v e)$ record which means distally inclined crown. The procedure was done for all the teeth of the dental arch right and left sides, upper and lower arches for both genders.

The data were subjected to statistical analysis using Minitab statistical program and this included: Descriptive statistics (means, standard deviation, minimum and maximum values), correlation of all the mesiodistal crowns angulation, and Student's t-test to differentiate between right and left, males and females. Results were considered as significant when $p \leq 0.05$.

\section{RESULTS AND DISCUSSION}

Tables (1) and (2) demonstrated the comparison between right-left sides teeth angulations of upper and lower dental arches for males and females, respectively. There was insignificant difference between right and left sides for both males and females and this result came in agreement with Al-Dewachi, ${ }^{(9)}$ although his study carried out on adults of Class I normal occlusion using panoramic radiograph. Bilateral symmetry also noted by Farah $^{(24)}$ in a study on the angulation of the developing maxillary canines and by Alwash ${ }^{(25)}$ in her study on the developmental position of mandibular canine, first, second premolars, first and second molars, so that the combined means (right and left) for the description of the variables and for comparison between males and females were taken.

Table (1): Comparison between right-left sides crowns angulations of males upper and lower dental arches in Class II division 1 malocclusion.

\begin{tabular}{|c|c|c|c|c|c|c|c|c|c|}
\hline \multirow{2}{*}{ Teeth } & \multirow{2}{*}{ Sid } & \multicolumn{4}{|c|}{ Upper } & \multicolumn{4}{|r|}{ Lower } \\
\hline & & Mean & $\pm \mathrm{SD}$ & t-value & Significance & Mean & \pm SD & t-value & Significance \\
\hline Central & $\begin{array}{l}\mathrm{R} \\
\mathrm{L}\end{array}$ & $\begin{array}{l}3.75 \\
2.85\end{array}$ & $\begin{array}{l}2.91 \\
3.27\end{array}$ & 1.24 & Not Significant & $\begin{array}{l}0.73 \\
0.63\end{array}$ & $\begin{array}{l}1.64 \\
1.65\end{array}$ & 0.22 & Not Significant \\
\hline Lateral & $\begin{array}{l}\mathrm{R} \\
\mathrm{L}\end{array}$ & $\begin{array}{l}6.58 \\
5.58\end{array}$ & $\begin{array}{l}4.30 \\
3.46\end{array}$ & 1.32 & Not Significant & $\begin{array}{l}0.70 \\
1.13\end{array}$ & $\begin{array}{l}1.41 \\
2.28\end{array}$ & -0.5 & Not Significant \\
\hline Canine & $\begin{array}{l}\mathrm{R} \\
\mathrm{L}\end{array}$ & $\begin{array}{l}5.75 \\
4.94\end{array}$ & $\begin{array}{l}4.28 \\
4.01\end{array}$ & 0.61 & Not Significant & $\begin{array}{l}-0.3 \\
0.87\end{array}$ & $\begin{array}{c}3.75 \\
4.1\end{array}$ & -0.99 & Not Significant \\
\hline $\begin{array}{c}\text { First } \\
\text { Premolar }\end{array}$ & $\begin{array}{l}\mathrm{R} \\
\mathrm{L}\end{array}$ & $\begin{array}{l}2.95 \\
2.48\end{array}$ & $\begin{array}{l}3.53 \\
3.05\end{array}$ & 0.39 & Not Significant & $\begin{array}{l}2.75 \\
2.38\end{array}$ & $\begin{array}{l}3.67 \\
4.45\end{array}$ & -0.98 & Not Significant \\
\hline $\begin{array}{l}\text { Second } \\
\text { Premolar }\end{array}$ & $\begin{array}{l}\mathrm{R} \\
\mathrm{L}\end{array}$ & $\begin{array}{l}1.89 \\
1.73\end{array}$ & $\begin{array}{l}3.01 \\
2.71\end{array}$ & 0.28 & Not Significant & $\begin{array}{l}2.53 \\
2.80\end{array}$ & $\begin{array}{l}2.44 \\
3.85\end{array}$ & -0.28 & Not Significant \\
\hline $\begin{array}{l}\text { First } \\
\text { Molar }\end{array}$ & $\begin{array}{l}\mathrm{R} \\
\mathrm{L}\end{array}$ & $\begin{array}{l}1.68 \\
1.28\end{array}$ & $\begin{array}{l}2.96 \\
2.56\end{array}$ & 0.42 & Not Significant & $\begin{array}{l}2.70 \\
3.77\end{array}$ & $\begin{array}{l}2.40 \\
3.99\end{array}$ & -0.94 & Not Significant \\
\hline
\end{tabular}

R: Right, L: Left, SD: Standard deviation. 
Table (2): Comparison between right-left sides crowns angulations of females upper and lower dental arches in Class II division 1 malocclusion

\begin{tabular}{|c|c|c|c|c|c|c|c|c|c|}
\hline \multirow{2}{*}{ Teeth } & \multirow{2}{*}{ Side } & \multicolumn{4}{|c|}{ Upper } & \multicolumn{4}{|c|}{ Lower } \\
\hline & & Mean & \pm SD & t-value & ice 1 & Mean & $\pm \mathrm{SD}$ & t-value & $\overline{\text { Significance }}$ \\
\hline \multirow[b]{2}{*}{ Centr } & $\mathrm{R}$ & 368 & 3.27 & \multirow{2}{*}{1.36} & \multirow{2}{*}{ Not Significant } & 10 & 1.65 & \multirow[b]{2}{*}{6} & \multirow{2}{*}{ Not Significant } \\
\hline & $\mathrm{L}$ & 2.38 & 2.28 & & & 1.75 & 1.61 & & \\
\hline \multirow{2}{*}{ Later } & $\mathrm{R}$ & 5.33 & 3.46 & \multirow{2}{*}{1.22} & \multirow{2}{*}{ Not Significant } & 0.83 & 2.28 & \multirow{2}{*}{.45} & \multirow{2}{*}{ Not } \\
\hline & $\mathrm{L}$ & 4.28 & 2.47 & & & 1.18 & 1.77 & & \\
\hline \multirow{2}{*}{ Canine } & $\mathrm{R}$ & 5.20 & 4.03 & \multirow{2}{*}{0.4} & \multirow{2}{*}{ Not Significant } & 1.4 & 4.1 & \multirow{2}{*}{0.5} & \multirow{2}{*}{ Not Sig } \\
\hline & $\mathrm{L}$ & 420 & 4.26 & & & 2 & 4.35 & & \\
\hline Firs & $\mathrm{R}$ & 2.23 & 3.05 & \multirow{2}{*}{0.3} & \multirow{2}{*}{ Not Significant } & 1.35 & 4.45 & \multirow{2}{*}{0.74} & \multirow{2}{*}{ Not Significant } \\
\hline Premolar & $\mathrm{L}$ & 1.93 & 2.69 & & & 1.85 & 3.41 & & \\
\hline Second & $\mathrm{R}$ & 2.75 & 3.68 & \multirow{2}{*}{1.4} & \multirow{2}{*}{ Not Significant } & 2.23 & 3.85 & \multirow{2}{*}{1.18} & \multirow{2}{*}{ Not Significant } \\
\hline Pren & $\mathrm{L}$ & & 1.25 & & & 2.30 & 3.88 & & \\
\hline & $\mathrm{R}$ & 1.6 & 2.56 & \multirow{2}{*}{1.1} & \multirow{2}{*}{ Not Significant } & 3.08 & 3.99 & \multirow{2}{*}{1.28} & \multirow{2}{*}{ Not Significant } \\
\hline Molar & $\mathrm{L}$ & 1.10 & 2.71 & & & 3.7 & 3.86 & & \\
\hline
\end{tabular}

R: Right, L: Left, SD: Standard deviation.

Table (3) for description of mesiodistal crowns angulations of upper dental arch revealed that the females have larger mean value for upper central incisors than males. Also the female have larger record (maximum) for this crown than the males. In Class II division 1 malocclusion there was an increase in the over jet, and soft tissue appeared to play a role in the determination of the final position of incisors if hypoton- ic muscles of upper lip then lead to proclination of incisors or if hypertonic muscle, stretched, upper lip will pushing the incisors palatally to yield division two. So, the hypotonic of female lip gave the higher records in the data and the mean value.

Also the females have slightly larger records for upper first molar than males. The males have larger records for the other crowns.

Table (3): Description of Mesio-distal crowns angulations of upper dental arch in Class II division1 malocclusion

\begin{tabular}{|c|c|c|c|c|c|}
\hline Teeth & Sex & Mean & $\pm \mathrm{SD}$ & Minimum & Maximum \\
\hline \multirow{3}{*}{ Central } & M & 2.41 & 2.91 & 0.0 & 9.0 \\
\hline & $\mathrm{F}$ & 3.01 & 3.27 & 0.0 & 13.0 \\
\hline & $\mathrm{T}$ & 2.91 & 2.81 & 0.0 & 13.0 \\
\hline \multirow{3}{*}{ Lateral } & M & 5.58 & 4.30 & 0.0 & 20.0 \\
\hline & $\mathrm{F}$ & 4.30 & 3.46 & 0.0 & 11.0 \\
\hline & $\mathrm{T}$ & 4.94 & 4.10 & 0.0 & 20.0 \\
\hline \multirow{3}{*}{ Canine } & M & 5.30 & 4.28 & 0.0 & 14.0 \\
\hline & $\mathrm{F}$ & 4.31 & 4.01 & 0.0 & 14.0 \\
\hline & $\mathrm{T}$ & 5.03 & 4.10 & 0.0 & 14.0 \\
\hline \multirow{3}{*}{$\begin{array}{c}\text { First } \\
\text { Premolar }\end{array}$} & M & 2.95 & 3.53 & -4.0 & 12.0 \\
\hline & F & 2.23 & 3.05 & 0.0 & 9.0 \\
\hline & $\mathrm{T}$ & 2.39 & 3.34 & -4.0 & 12.0 \\
\hline \multirow{3}{*}{$\begin{array}{l}\text { Second } \\
\text { Premolar }\end{array}$} & M & 1.98 & 3.01 & -2.0 & 11.0 \\
\hline & $\mathrm{F}$ & 0.63 & 2.71 & 0.0 & 15.0 \\
\hline & $\mathrm{T}$ & 1.77 & 2.84 & -2.0 & 15.0 \\
\hline \multirow{3}{*}{$\begin{array}{l}\text { First } \\
\text { Molar }\end{array}$} & M & 1.58 & 2.96 & -2.0 & 13.0 \\
\hline & $\mathrm{F}$ & 1.68 & 2.56 & -7.0 & 9.0 \\
\hline & $\mathrm{T}$ & 1.18 & 2.85 & -7.0 & 13.0 \\
\hline
\end{tabular}

SD: Standard deviation, M: Male, F: Female, T: Total. 
Table (4) for description of crowns angulation for lower dental arch showed that males have slightly larger mean value for second premolar and larger records (maximum) than females. For the other crowns, females have larger records. There was no previous researches taking the mesiodistal crowns angulation directly from the study casts. All the studies measured this variable on $\mathrm{x}$-ray films, usually they take panoramic films, and mesured the mesiodistal angulation with the occlusal plane, so the record values round $90^{\circ}$. In Mosul City, only Al-Dewachi ${ }^{(9)}$ research took mesiodistal angulation, but he took panoramic films, and the sample on adult patients having Class I normal occlusion. This research is considered the first one on measuring the mesiodistal angulation directly on study models and on Class II division 1 malocclusion.

Table (4): Description of Mesio-distal crowns angulations of lower dental arch in Class II division1 malocclusion

\begin{tabular}{cccccc}
\hline Teeth & Sex & Mean & \pm SD & Minimum & Maximum \\
\hline \multirow{4}{*}{ Central } & M & 0.73 & 1.64 & 0.0 & 6.0 \\
& $\mathrm{~F}$ & 1.1 & 1.65 & 0.0 & 7.0 \\
& $\mathrm{~T}$ & 1.53 & 1.63 & 0.0 & 7.0 \\
Lateral & $\mathrm{M}$ & 0.70 & 1.41 & -13.0 & 7.0 \\
& $\mathrm{~F}$ & 0.83 & 2.28 & -13.0 & 7.0 \\
& $\mathrm{~T}$ & 0.74 & 2.88 & -13.0 & 7.0 \\
Canine & $\mathrm{M}$ & -0.3 & 3.75 & -13.0 & 8.5 \\
& $\mathrm{~F}$ & 1.4 & 4.1 & -4.0 & 15.0 \\
& $\mathrm{~T}$ & 0.96 & 3.88 & -13.0 & 15.0 \\
First & $\mathrm{M}$ & 0.87 & 3.67 & -4.0 & 10.0 \\
Premolar & $\mathrm{F}$ & 1.35 & 4.45 & -1.0 & 13.0 \\
& $\mathrm{~T}$ & 1.69 & 3.61 & -4.0 & 13.0 \\
Second & $\mathrm{M}$ & 2.53 & 2.44 & 0.0 & 10.0 \\
Premolar & $\mathrm{F}$ & 2.30 & 3.85 & -12.0 & 14.0 \\
& $\mathrm{~T}$ & 2.18 & 3.04 & -12.0 & 14.0 \\
First & $\mathrm{M}$ & 2.7 & 2.40 & 0.0 & 14.0 \\
Molar & $\mathrm{F}$ & 3.08 & 3.99 & 0.0 & 13.0 \\
& $\mathrm{~T}$ & 2.58 & 3.46 & 0.0 & 14.0 \\
\hline
\end{tabular}

SD: Standard deviation, M: Male, F: Female, T: Total.

In Table (5), comparison between sexes for each quadrant was done and no significant difference was found. Similar results were found in the Tables (6) and (7) for comparison of combined (right-left) sides crowns angulations between males-females upper and lower dental arches respectively and revealed no significant differences between sexes for upper and lower dental arches. This result came in accordance with Al-Dewachi, ${ }^{(9)}$ who found no significant difference in the mesiodistal crowns angulation between the two sexes except for first mandibular premolar and first mandibular molar and this difference may be due to difference in sample age, occlusion type and method of measurement.

Table (8) showed the correlation of the teeth in the upper dental arch. A high correlation between (lateral-canine) and (second premolar-first molar) was found. A moderate correlation was found between (central-lateral), (canine-first premolar), (first-second premolars), (first premolarfirst molar). From these relations, it could be concluded that all the teeth correlated with each other; an increase in the angulations of one segment of the dental arch will be accompanied by an increase in the angulation in the other segment. 
Table (5): Comparison of crowns angulation between male and female for right and left sides in upper and lower dental arch in Class II division 1 malocclusion

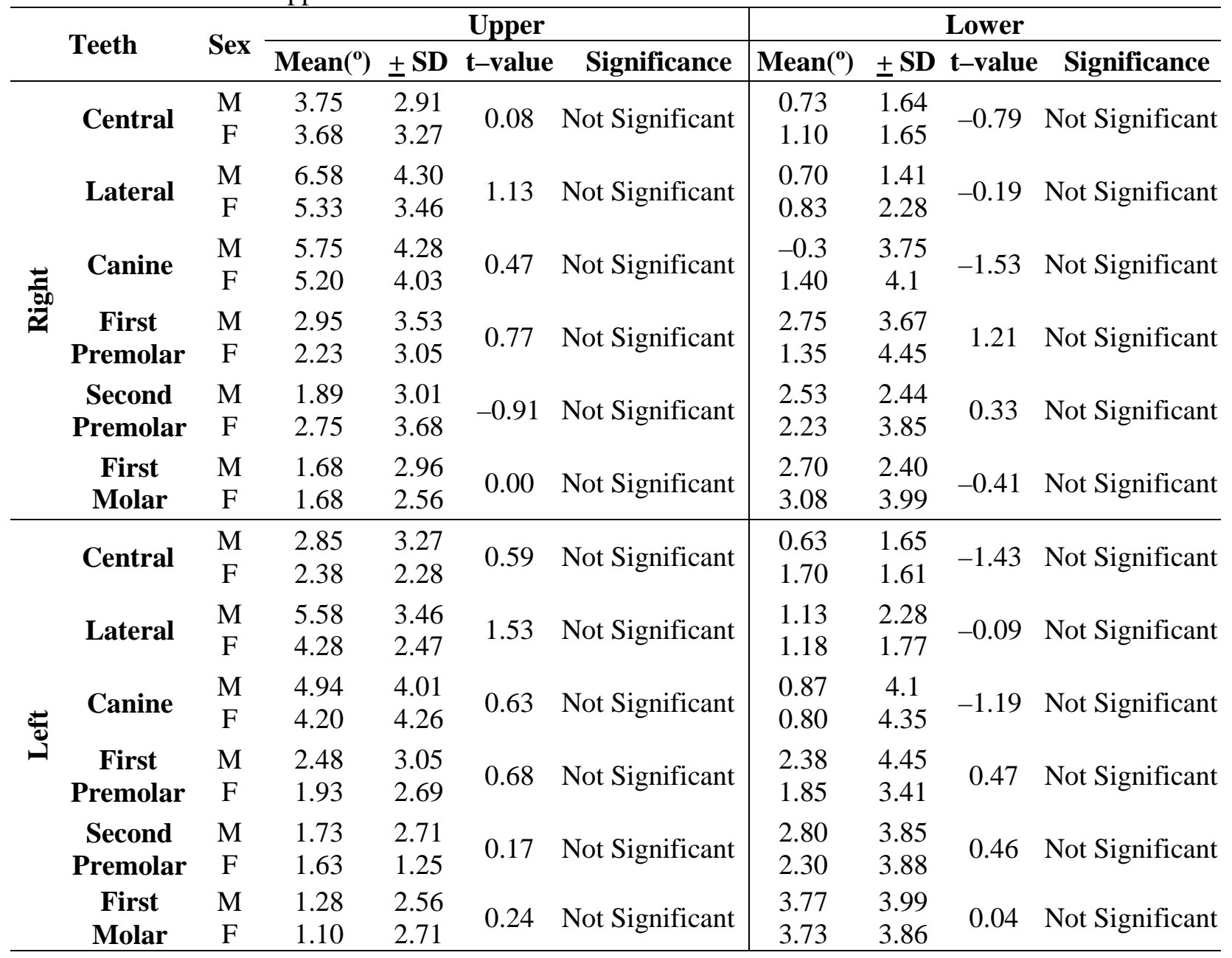

SD: Standard deviation, M: Male, F: Female.

Table (6): Comparison of combined right-left sides crowns angulations of males-females upper dental arches in Class II division 1 malocclusion

\begin{tabular}{cccccc}
\hline Teeth & Sex & Mean & \pm SD & t-value & Significance \\
\hline \multirow{2}{*}{ Central } & M & 2.41 & 2.91 & & \\
& F & 3.01 & 3.27 & -0.35 & Not Significant \\
Lateral & M & 5.58 & 4.30 & & \\
& F & 4.30 & 3.46 & 1.40 & Not Significant \\
Canine & M & 5.30 & 4.28 & & \\
& F & 4.31 & 4.01 & 0.7 & Not Significant \\
First & M & 2.95 & 3.53 & & \\
Premolar & F & 2.23 & 3.05 & 0.85 & Not Significant \\
Second & M & 1.98 & 3.01 & & \\
Premolar & F & 0.63 & 2.71 & 0.25 & Not Significant \\
First & M & 1.58 & 2.96 & & \\
Molar & F & 1.68 & 2.56 & 0.92 & Not Significant \\
\hline
\end{tabular}

SD: Standard deviation, M: Male, F: Female. 
Table (7): Comparison of combined right-left sides crowns angulations of males-females lower dental arches in Class II division 1 malocclusion

\begin{tabular}{|c|c|c|c|c|c|}
\hline Teeth & Sex & Mean & \pm SD & t-value & Significance \\
\hline Central & $\begin{array}{c}M \\
F\end{array}$ & & & -3.07 & Significant \\
\hline Lateral & $\begin{array}{c}\mathrm{M} \\
\mathrm{F}\end{array}$ & $\begin{array}{l}0.70 \\
0.83\end{array}$ & $\begin{array}{l}1.41 \\
2.28\end{array}$ & 0.54 & Not Significant \\
\hline Canine & $\begin{array}{l}\mathrm{M} \\
\mathrm{F}\end{array}$ & $\begin{array}{c}-0.3 \\
1.4\end{array}$ & $\begin{array}{c}3.75 \\
4.1\end{array}$ & & ificant \\
\hline First Premolar & $\begin{array}{l}\text { M } \\
\mathrm{F}\end{array}$ & $\begin{array}{l}2.75 \\
1.35\end{array}$ & $\begin{array}{l}3.67 \\
4.45\end{array}$ & -0.32 & Not Significant \\
\hline Second Premolar & $\begin{array}{l}\mathrm{M} \\
\mathrm{F}\end{array}$ & $\begin{array}{l}2.53 \\
2.23\end{array}$ & $\begin{array}{l}2.4 \\
3.8\end{array}$ & 1.43 & Not Significant \\
\hline First Molar & $\begin{array}{c}\mathrm{M} \\
\mathrm{F} \\
\end{array}$ & $\begin{array}{c}2.7 \\
3.08 \\
\end{array}$ & $\begin{array}{l}2.40 \\
3.99 \\
\end{array}$ & 1.73 & Not Significant \\
\hline
\end{tabular}

SD: Standard deviation, M: Male, F: Female.

Table (8): Correlation of crowns angulations of upper dental arch in Class II division1 malocclusion

\begin{tabular}{ccccccc}
\hline Teeth & Sex & Central & Lateral & Canine & $\begin{array}{c}\text { First } \\
\text { Premolar Premolar }\end{array}$ \\
\hline \multirow{4}{*}{ Lateral } & M & 0.50 & & & & \\
& $\mathrm{~F}$ & 0.44 & & & & \\
& $\mathrm{~T}$ & 0.46 & & & & \\
& $\mathrm{M}$ & 0.13 & 0.53 & & & \\
Canine & $\mathrm{F}$ & 0.21 & 0.69 & & & \\
& $\mathrm{~T}$ & 0.22 & 0.61 & & & \\
First & $\mathrm{M}$ & -0.06 & 0.20 & 0.40 & & \\
Premolar & $\mathrm{F}$ & -0.19 & 0.16 & 0.42 & & \\
& $\mathrm{~T}$ & -0.14 & 0.20 & 0.41 & & \\
Second & $\mathrm{M}$ & -0.18 & -0.06 & 0.12 & 0.41 & \\
Premolar & $\mathrm{F}$ & 0.14 & 0.18 & 0.08 & 0.44 & \\
& $\mathrm{~T}$ & 0.16 & 0.12 & 0.09 & 0.43 & \\
First & $\mathrm{M}$ & -0.13 & 0.04 & 0.29 & 0.45 & 0.73 \\
Molar & $\mathrm{F}$ & 0.02 & 0.34 & 0.31 & 0.41 & 0.67 \\
& $\mathrm{~T}$ & 0.13 & 0.16 & 0.30 & 0.43 & 0.70 \\
\hline
\end{tabular}

M: Male, F: Female, T: Total

Similarly, Table (9) revealed the correlation of the teeth in the lower dental arch. A moderate correlation was found between (central-lateral), (lateral-canine), (first premolar with both lateral and canine), (first premolar-first molar), while in Table (10) for correlation of upper and lower dental arches a weak correlation existed among upper and lower crowns.

From these three Tables (8-10) it could be noticed that there was a correlation between the teeth in the anterior segment and there was a correlation between the te- eth in the posterior segment but less correlation was present between the anterior and posterior segment. There was a significant intraarch correlation, a moderate correlation between crowns in the upper arch and in the lower arch but there was insignificant interarch correlation. This may be related to the position of the teeth within the basal bone. In case of Class II division 1 malocclusion in which the cause of malocclusion may be skeletal in the maxilla or the mandible and not to dental cause, that is why the teeth will affected by the grow- 
th pattern of its basal bone.

In Table (11) comparison between Class I-Class II crowns angulations of males in upper and lower dental arches was noticed. There was a significant difference in the buccal segment of upper arch (canine, first and second premolars and first molar) that the mean value was larger in Class II than in Class I which means more mesially angulated crowns in Class II. This gives idea that the cause of this malocclusion is the dental arch. For the lower arch there was significant difference for central, lateral and the second premolar. From these results, it could be noted that the upper and lower crowns were more mesially angulat-ed in Class II division 1 malocclusion.

Table (9): Correlation of crowns angulations of lower dental arch in Class II division 1 malocclusion

\begin{tabular}{ccccccc}
\hline Teeth & Sex & Central & Lateral & Canine & $\begin{array}{c}\text { First } \\
\text { Premolar Premolar }\end{array}$ \\
\hline \multirow{2}{*}{ Lateral } & Male & 0.44 & & & & \\
& Female & -0.34 & & & & \\
& Total & -0.39 & & & & \\
Canine & Male & 0.24 & 0.42 & & & \\
& Female & 0.08 & 0.38 & & & \\
& Total & 0.16 & 0.40 & & & \\
First & Male & 0.07 & 0.44 & 0.36 & & \\
Premolar & Female & 0.12 & 0.49 & 0.57 & & \\
& Total & 0.10 & 0.47 & 0.46 & & \\
Second & Male & -0.12 & 0.12 & 0.28 & 0.38 & \\
Premolar & Female & -0.21 & 0.12 & 0.26 & 0.27 & \\
& Total & -0.14 & 0.12 & 0.27 & 0.32 & \\
First & Male & -0.19 & 0.12 & 0.15 & 0.26 & 0.55 \\
Molar & Female & -0.07 & 0.08 & 0.05 & 0.12 & 0.45 \\
& Total & -0.20 & 0.09 & 0.08 & 0.15 & 0.50 \\
\hline
\end{tabular}

Table (10): Correlation of crowns angulations of upper-lower dental arch in Class II division 1 malocclusion

\begin{tabular}{|c|c|c|c|c|c|c|c|c|}
\hline & \multirow[b]{2}{*}{ Teeth } & \multirow[b]{2}{*}{ Sex } & \multicolumn{6}{|c|}{ Upper } \\
\hline & & & Central & Lateral & Canine & $\begin{array}{c}\text { First } \\
\text { Premolar }\end{array}$ & $\begin{array}{l}\text { Second } \\
\text { Premolar }\end{array}$ & $\begin{array}{l}\text { First } \\
\text { Molar }\end{array}$ \\
\hline \multirow{15}{*}{ 离 } & \multirow{3}{*}{ Central } & Male & -0.0 & -0.1 & 0.23 & 0.17 & 0.21 & -0.03 \\
\hline & & Female & -0.29 & -0.4 & -0.18 & 0.01 & -0.19 & -0.5 \\
\hline & & Total & -0.15 & -0.2 & -0.20 & 0.09 & -0.20 & -0.21 \\
\hline & \multirow{3}{*}{ Lateral } & Male & 0.05 & 0.18 & 0.37 & 0.18 & 0.18 & 0.21 \\
\hline & & Female & -0.07 & 0.05 & -0.04 & 0.26 & -0.01 & 0.10 \\
\hline & & Total & -0.06 & 0.11 & -0.21 & 0.22 & -0.90 & 0.16 \\
\hline & \multirow{3}{*}{ Canine } & Male & 0.01 & 0.11 & -0.44 & -0.03 & -0.15 & -0.18 \\
\hline & & Female & -0.35 & 0.10 & 0.18 & -0.14 & -0.25 & 0.25 \\
\hline & & Total & -0.20 & 0.10 & -0.31 & -0.08 & -0.20 & -0.22 \\
\hline & \multirow{3}{*}{$\begin{array}{c}\text { First } \\
\text { Premolar }\end{array}$} & Male & -0.32 & 0.01 & 0.06 & 0.37 & 0.12 & 0.18 \\
\hline & & Female & -0.35 & 0.16 & 0.07 & 0.19 & -0.19 & 0.10 \\
\hline & & Total & -0.33 & 0.04 & 0.06 & 0.28 & -0.15 & 0.14 \\
\hline & \multirow{3}{*}{$\begin{array}{c}\text { Second } \\
\text { Premolar }\end{array}$} & Male & -0.10 & -0.02 & 0.05 & 0.05 & 0.01 & -0.05 \\
\hline & & Female & -0.17 & 0.13 & -0.03 & 0.25 & -0.01 & 0.21 \\
\hline & & Total & -0.13 & -0.07 & -0.04 & 0.15 & 0.01 & -0.13 \\
\hline
\end{tabular}




\begin{tabular}{cccccccc} 
First & Male & -0.24 & v0.1 & 0.13 & 0.04 & 0.33 & 0.34 \\
Molar & Female & -0.12 & 0.4 & 0.24 & 0.27 & 0.08 & 0.07 \\
& Total & -0.20 & 0.3 & 0.16 & 0.17 & 0.16 & 0.21 \\
\hline
\end{tabular}

Table (11): Comparison between Class I-Class II crowns angulations of males upper and lower dental arches

\begin{tabular}{|c|c|c|c|c|c|c|c|c|c|}
\hline \multirow{2}{*}{ Teeth } & \multirow{2}{*}{ Class } & Mean & \pm SD & t-value & Significance & Mean & $\pm \mathrm{SD}$ & t-value & Significance \\
\hline & & \multicolumn{4}{|c|}{ Upper } & \multicolumn{4}{|c|}{ Lower } \\
\hline Central & $\begin{array}{l}\text { I } \\
\text { II }\end{array}$ & $\begin{array}{l}2.72 \\
2.41\end{array}$ & $\begin{array}{l}2.26 \\
2.91\end{array}$ & 0.12 & $\begin{array}{c}\text { Not } \\
\text { Significant }\end{array}$ & $\begin{array}{c}-1.21 \\
0.73\end{array}$ & $\begin{array}{l}1.38 \\
1.65\end{array}$ & 5.95 & Significant \\
\hline Lateral & $\begin{array}{l}\text { I } \\
\text { II }\end{array}$ & $\begin{array}{l}5.62 \\
5.58\end{array}$ & $\begin{array}{l}3.29 \\
4.30\end{array}$ & & $\begin{array}{c}\text { Not } \\
\text { Significant }\end{array}$ & $\begin{array}{l}-1.50 \\
0.70\end{array}$ & $\begin{array}{l}2.09 \\
1.41\end{array}$ & 5 & Sig \\
\hline Canine & $\begin{array}{l}\text { I } \\
\text { II }\end{array}$ & $\begin{array}{l}2.97 \\
5.30\end{array}$ & $\begin{array}{l}3.21 \\
4.28\end{array}$ & 2.70 & Significant & $\begin{array}{l}1.07 \\
-0.3\end{array}$ & $\begin{array}{l}2.87 \\
3.75\end{array}$ & -0.99 & $\begin{array}{c}\text { Not } \\
\text { Significant }\end{array}$ \\
\hline $\begin{array}{c}\text { First } \\
\text { Premolar }\end{array}$ & $\begin{array}{l}\text { I } \\
\text { II }\end{array}$ & $\begin{array}{l}1.17 \\
2.95\end{array}$ & $\begin{array}{l}1.98 \\
3.53\end{array}$ & 2.19 & Significant & $\begin{array}{l}0.19 \\
0.87\end{array}$ & $\begin{array}{l}2.53 \\
3.67\end{array}$ & .98 & $\begin{array}{c}\text { Not } \\
\text { Significant }\end{array}$ \\
\hline $\begin{array}{l}\text { Second } \\
\text { Premolar }\end{array}$ & $\begin{array}{l}\text { I } \\
\text { II }\end{array}$ & $\begin{array}{l}0.62 \\
1.98\end{array}$ & $\begin{array}{l}2.03 \\
3.01\end{array}$ & 2.12 & Significant & $\begin{array}{l}0.33 \\
2.53\end{array}$ & $\begin{array}{l}1.70 \\
2.44\end{array}$ & 3.81 & Significant \\
\hline $\begin{array}{l}\text { First } \\
\text { Molar }\end{array}$ & $\begin{array}{l}\text { I } \\
\text { II }\end{array}$ & $\begin{array}{l}0.21 \\
1.58\end{array}$ & $\begin{array}{l}3.13 \\
2.96\end{array}$ & 1.99 & Significant & $\begin{array}{c}1.95 \\
2.7\end{array}$ & $\begin{array}{l}3.13 \\
2.40\end{array}$ & 1.31 & $\begin{array}{c}\text { Not } \\
\text { Significant }\end{array}$ \\
\hline
\end{tabular}

SD: Standard deviation.

Similar results were found for females as could be seen in Table (12) for comparison between Class I-Class II crowns angulations of females. In the upper dental arches, there was significant difference for buccal teeth like in males except for the central and lateral incisors. The difference was insignificant, but the mean values were larger in Class II than in Class I. For the lower crowns there was significant difference for all the crowns except for first mol- ar which was insignificant. The mean values for crowns angulation were higher in Class II malocclusion than in Class I sample of this research. Also, it was higher than the values found by Al-Dewachi ${ }^{(9)}$ for Class I normal occlusion and higher than the documented values for brackets angulation established by other studies. ${ }^{(26-28)}$ This supports the results of this study that the teeth were more mesially angulated in Class II malocclusion.

Table (12): Comparison between Class I-Class II crowns angulations of females upper and lower dental arches

\begin{tabular}{|c|c|c|c|c|c|c|c|c|c|}
\hline \multirow{2}{*}{ Teeth } & \multirow{2}{*}{ Class } & Mean & $\pm \mathrm{SD}$ & t-value & Significance & Mean & \pm SD & t-value & Significance \\
\hline & & \multicolumn{4}{|c|}{ Upper } & \multicolumn{4}{|c|}{ Lower } \\
\hline Central & $\begin{array}{l}\text { I } \\
\text { II }\end{array}$ & $\begin{array}{l}3.11 \\
3.01\end{array}$ & $\begin{array}{l}1.25 \\
3.27\end{array}$ & 0.98 & $\begin{array}{c}\text { Not } \\
\text { Significant }\end{array}$ & $\begin{array}{c}-0.01 \\
1.10\end{array}$ & $\begin{array}{l}1.38 \\
1.65\end{array}$ & 3.95 & Significant \\
\hline Lateral & $\begin{array}{c}\text { I } \\
\text { II }\end{array}$ & $\begin{array}{l}4.31 \\
4.30\end{array}$ & $\begin{array}{l}2.53 \\
3.46\end{array}$ & -1.05 & $\begin{array}{c}\text { Not } \\
\text { Significant }\end{array}$ & $\begin{array}{c}-0.26 \\
0.83\end{array}$ & $\begin{array}{l}1.95 \\
2.28\end{array}$ & 4.95 & Significant \\
\hline Canine & $\begin{array}{c}\text { I } \\
\text { II }\end{array}$ & $\begin{array}{l}1.30 \\
4.31\end{array}$ & $\begin{array}{l}1.36 \\
4.01\end{array}$ & 3.66 & Significant & $\begin{array}{l}1.07 \\
1.40\end{array}$ & $\begin{array}{l}0.86 \\
4.10\end{array}$ & 2.11 & $\begin{array}{c}\text { Not } \\
\text { Significant }\end{array}$ \\
\hline $\begin{array}{c}\text { First } \\
\text { Premolar }\end{array}$ & $\begin{array}{c}\text { I } \\
\text { II }\end{array}$ & $\begin{array}{l}0.74 \\
2.23\end{array}$ & $\begin{array}{l}1.27 \\
3.05\end{array}$ & 2.43 & Significant & $\begin{array}{l}0.19 \\
1.35\end{array}$ & $\begin{array}{l}2.11 \\
4.45\end{array}$ & 2.40 & $\begin{array}{c}\text { Not } \\
\text { Significant }\end{array}$ \\
\hline $\begin{array}{l}\text { Second } \\
\text { Premolar }\end{array}$ & $\begin{array}{c}\text { I } \\
\text { II }\end{array}$ & $\begin{array}{l}0.57 \\
2.63\end{array}$ & $\begin{array}{l}1.58 \\
2.71\end{array}$ & 2.58 & Significant & $\begin{array}{l}0.46 \\
2.30\end{array}$ & $\begin{array}{l}0.70 \\
3.85\end{array}$ & 3.82 & Significant \\
\hline
\end{tabular}




\begin{tabular}{cccccc|cccc} 
First & I & -3.90 & 3.06 & \multirow{2}{*}{6.93} & \multirow{2}{*}{ Significant } & 0.34 & 2.52 & \multirow{2}{*}{1.31} & Not \\
Molar & II & 1.68 & 2.56 & & & & & \\
Significant
\end{tabular}

SD: Standard deviation.

\section{CONCLUSION}

The crowns were more mesially angulated in Class II division malocclusion and this fact must be taken in consideration during correction of this type of malocclusion.

All the crowns correlated with each other, an increase in the angulation of one segment of the dental arch will affect on the other segment.

\section{REFERENCES}

1. Tang EL. The prevalence of malocclusi-on among Hong Kong male dental stude-nts. $\mathrm{Br} J$ Orthod. 1994, 21: 57-68.

2. Lauc TO. Facial analysis on the Adriatic Islands: An epidemiological study of ma-locclusion on Hrar Island. Eur J Orthod. 2001; 23: 273278.

3. Onyeaso CO, Aderinokum GA, Arowoj-olu MO. The pattern of malocclusion among orthodontic patient seen in dental center, University College Hospital, Ibadan, Nigeria. Afr J Med Sci. 2002; 31: 207-211.

4. Tausche E, Luck O, Harzer W. Prevalen-ce of malocclusion in the early mixed de-ntition and orthodontic treatment need. Eur $J$ Orthod. 2004; 26: 237-244.

5. Sonnesen L, Bakke M, Solow B. Bite fo-rces in pre-orthodontic children with un-ilateral cross bite. Eur J Orthod. 2002; 24: 442-443.

6. Jassim FY. Prevalence of malocclusion in Mosul City 12-14 years old children. Accepted for publication in Iraqi Dent J. 2001.

7. Khamarco TY, Al-Khatib AR, Agha NF. Occlusal criteria in two Iraqi rural communities. Al-Rafidain Dent J. 2002; 2: 360-368.

8. Andrews LF. The six keys to normal oc-clusion. Am J Orthod. 1972; 62: 296-309.

9. Al-Dewachi ZB. Mesiodistal axial teeth inclination of permanent teeth in Mosul City in 18-25 year old in different third molar position in Class I normal occlusi-on (orthopantomographic study). MSc thesis. College of Dentistry. University of Mosul. 1999.

10. Dewel BF. Clinical observations on the axial inclination of teeth. Am $J$ Orthod. 1949; 35: 296-309.

11. Husseles W. Nanda RS. Effect of maxill-ary incisor angulation and inclination on arch length. Am $J$ Orthod Dentofac Orth-op. 1989; 95: 233-239.

12. Wheeler RC. Dental anatomy, Physiolo-gy and Occlusion. $7^{\text {th }}$ ed. WB Saunders Co. Philadelphia. 1993; Pp: 423-428.

13. Lucchesi MV, Wood RE, Nortje CJ. Sui-tability of panoramic radiograph for ass-essment of mesiodistal angulation of tee-th in the buccal segment of the mandible. Am $J$ Orthod Dentofac Orthop. 1988; 94: 303-310.

14. Kattner PF, Schneider BJ. Comparison of Roth appliance and standard edgewise appliance treatment results. Am J Orthod Dentofac Orthop. 1993; 103: 24-32.

15. Kinaan BK. Clinical evaluation of malp-osed canine. Iraqi Dent $J$. 1994; 17: 83-89.

16. Kinaan BK. Simple combined dental lat-he and model trimming machine. Iraqi Dent J. 1990; 15: 4-10.

17. Hatasaka HH. A radiographic study of roots in extraction sites. Angle Orthod. 1976; 46: 64-68.

18. Richardson ME. Pre-eruptive movemen-ts of mandibular third molar. Angle Orth-od. 1978; 48: 178-193.

19. McKee W, Williamson PC, Lam EW, Heo G, Glover KE, Major PW. The acc-uracy of four panoramic units in the proj-ection of mesiodistal tooth angulation. Am J Orthod Dentofac Orthop. 2002; 121: 166173. 
20. Al-Saleem NR. Differential diagnosis of Class II division 1 malocclusion (A cep-halometric study). MSc thesis. College of Dentistry. University of Mosul. 2002.

21. Al-Sammak SM. Influence of craniofac-ial parameters on the mandible in Class II division 1 malocclusion (Three dimen-sional cephalometric study). MSc thesis. College of Dentistry. University of Mos-ul. 2005.

22. Ahmed MKh. Comparison between Cla-ss I normal occlusion and Class II divisi-on 1 malocclusion (A cephalometric stu-dy). MSc thesis. College of Dentistry. University of Mosul. 2002.

23. Al-Sultan MM. Cranial base parameters influence on the nasomaxillary complex in Class II division 1 malocclusion (Thr-ee dimensional cephalometric study). MSc thesis. College of Dentistry.
Unive-rsity of Mosul. 2005.

24. Farah ME. The orthodontic examination of children aged 9 and 10 years from Ba-ghdad, Iraq. MSc thesis. College of Den-tistry. University of Baghdad. 1988.

25. Alwash NA. A radiographic study of de-velopment and mesiodistal axial inclinat-ion of five mandibular teeth in 8-12 year old children. MSc thesis. College of De-ntistry. University of Baghdad. 1994.

26. Roth RH. Treatment mechanics for the straight wire appliance. In: Graber LW, Swain BF (eds). Othodontics: Current Principles and Techniques. $1^{\text {st }}$ ed. CV Mosby Co. St Luis. 1985.

27. Ricketts RM. Provocations and percepti-ons in craniofacial orthopedics: Book one. Part II. Denver: RMO Inc. 1989.

28. Hilgers JJ. Biprogressive simplified. Part II: The linear dynamic system. $J$ Clin Or-thod. 1987; 21: 716-734. 